\title{
Nonadiabatic resonances in a noisy Fitzhugh-Nagumo neuron model
}

\author{
S. Ripoll Massanés* and C. J. Pérez Vicente ${ }^{\dagger}$ \\ Departament de Física Fonamental, Facultat de Física, Universitat de Barcelona, Diagonal 647, 08028 Barcelona, Spain
}

(Received 23 July 1998; revised manuscript received 12 October 1998)

\begin{abstract}
We have analyzed the response of a noisy Fitzhugh-Nagumo neuronlike model (FN) to subthreshold external stimuli. In contrast to previous studies we have focused our attention on high-frequency signals which could be of interest for real systems such as nervous fibers in the auditory system. We show that the noisy FN behaves as a stochastic oscillator with a characteristic time scale whose effects remain in a wide range of situations. In the nonadiabatic regime of frequencies considered in this work we report several resonant behaviors which resemble those of classical deterministic oscillators but never the typical stochastic resonance phenomenon so often observed for low-frequency signals. [S1063-651X(99)01304-5]
\end{abstract}

PACS number(s): 87.19.La, 87.10.+e, 05.40.-a, 05.20.-y

\section{INTRODUCTION}

The analysis of the time-dependent properties of systems made up of excitable units is essential to understand the basic mechanisms that govern their dynamical evolution. It is also the natural bridge that allows one to compare experimental data with results obtained from theoretical models. Neural systems are perhaps the most representative and studied examples. Any realistic model aimed at reproducing the typical behavior of an ensemble of neurons or just an isolated unit must take into account several observed features. One of them is the high degree of variability or irregularity in the spike trains [1]. An appropriate balance between excitation and inhibition is required to achieve it, otherwise the interspike interval (ISI) distribution displays a nonrealistic regularity $[2,3]$. Moreover, it helps to keep the system firing with a low level of activity as well as to maintain a spontaneous activity even in the absence of external stimuli [4].

Noise is another factor that must be incorporated in any realistic description of excitable systems. Stochasticity, due to the Brownian motion of ions in a temperature bath, conductance changes induced by the random opening and closing of ion channels or simply because of the random reliability of synaptic buttons, just to cite some reported examples, play a relevant role in neural signal transmission. However, it would be wrong to think of an intrinsic source of randomness as an ingredient that could systematically worsen the computational properties of real systems. It is well known that the cooperation between noise and signal may induce a positive effect on the global features of nonlinear units by improving their ability to detect external subthreshold signals. Furthermore, the strong dependence of the ISI histogram shape on the noise strength indicates its importance when determining the variability of the firing pattern.

Recently, several authors have shown that without external inputs coherence in the response of a neuron model is maximized for an optimal value of the intensity of noise [5-7]. Such coherence or regularity defines a characteristic

\footnotetext{
*URL: http://www.ffn.ub.es/ sripoll/ Electronic address: sripoll@ffn.ub.es

†Electronic address: conrad@ffn.ub.es
}

time scale that always underlies and does affect the dynamics of the unit even in the presence of continuous stimuli. Notice that a high degree of coherence could be against the aforementioned variability since it may give rise to an undesirable robustness, preventing the system from inducing changes in the firing rate and as a consequence to transmit little information from the environment. In fact, in this context the process of maximizing the transferred information is equivalent to minimizing the overlap between signal and background spike distributions [5].

The goal of this paper is to analyze in detail these effects for a Fitzhugh-Nagumo (FN) neuronlike model. We have chosen this system because there is a large number of recent works considering its noisy response in front of either periodic and nonperiodic inputs and in this way we can make a comparison with them. We will determine the characteristic time of the system derived from the maximal observed level of coherence and we will establish also a relationship with the concept of stochastic resonance (SR) [8]. In contrast to many recent studies [9], we are not interested in the lowfrequency limit where signals are so slow that an adiabatic approximation can be applied to get analytic results. We focus our attention on a more realistic situation, in the effect of high-frequency signals, comparable with the intrinsic period of the model, whose analysis may be relevant for some real systems such as the auditory system [10].

The paper is structured as follows. Section II is devoted to the formal description of the model. In Sec. III we analyze the stochastic FN oscillator and determine the typical period of the system. The response of the model to high-frequency external stimuli is considered in Sec. IV. Finally, there is a short section devoted to conclusions and future work.

\section{THE MODEL}

In this paper we have considered a standard FN model whose dynamical behavior is governed by the following differential equations:

$$
\epsilon \frac{d v}{d t}=v(v-a)(1-v)-w+I,
$$



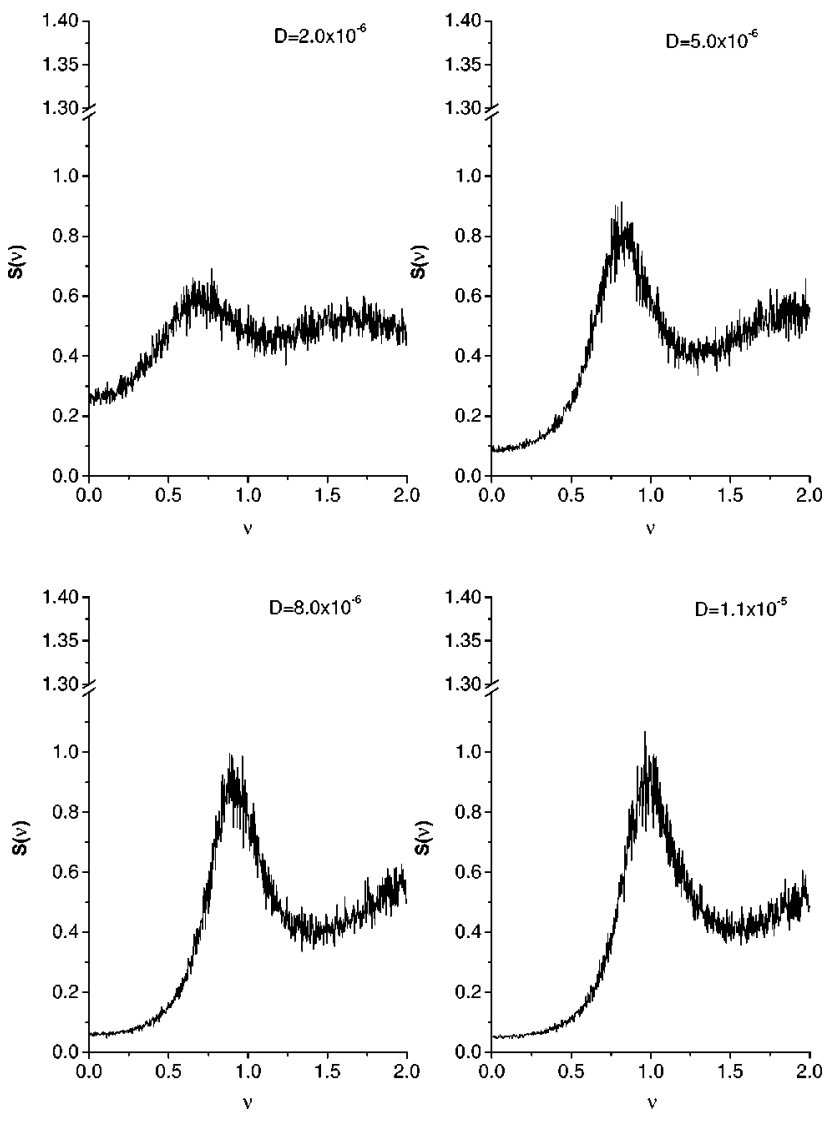

FIG. 1. Power spectrum for several values of $D$ for the pure stochastic FN neuron without external stimulus.

$$
\frac{d w}{d t}=v-p w-b
$$

where $\epsilon$ fixes the time scale of the fast variable $v$ (voltage), $w$ is the slow (recovery) variable, $I$ represents an external applied current, and $a, b$, and $p$ are constants whose values determine the stability properties of the system. Furthermore, a noise term $\gamma(t)$ can be added to the right-hand side of Eq. (1) as well as a forcing term $F(t)$. For an extensive analysis of the dynamic behavior of the model, see $[11,12]$. Throughout the paper we have fixed the values of the constants to $a=0.5, b=0.15, p=1.0$, and $\epsilon=0.005$ in order to compare our results with other previous studies on the same model [9,13-15]. The current $I$ will also be considered constant. In the deterministic case a subthreshold $I$ leads the system to a fixed point located at a well-controlled distance from the firing point. This is an approximate way to mimic experimental observed facts performed in vivo where it has been shown that, after firing, neurons return very quickly to an operative point very close to the threshold [16]. In this way, individual neurons have the ability to switch very fast from a resting state to an excited state in front of a selective external stimulus. For a suprathreshold constant current the system behaves as an oscillator.

\section{COHERENCE INDUCED BY NOISE}

In this section we analyze the effect of noise on the timedependent properties of the system. At this stage no deterministic forcing term $F(t)$ is considered, only a subthreshold
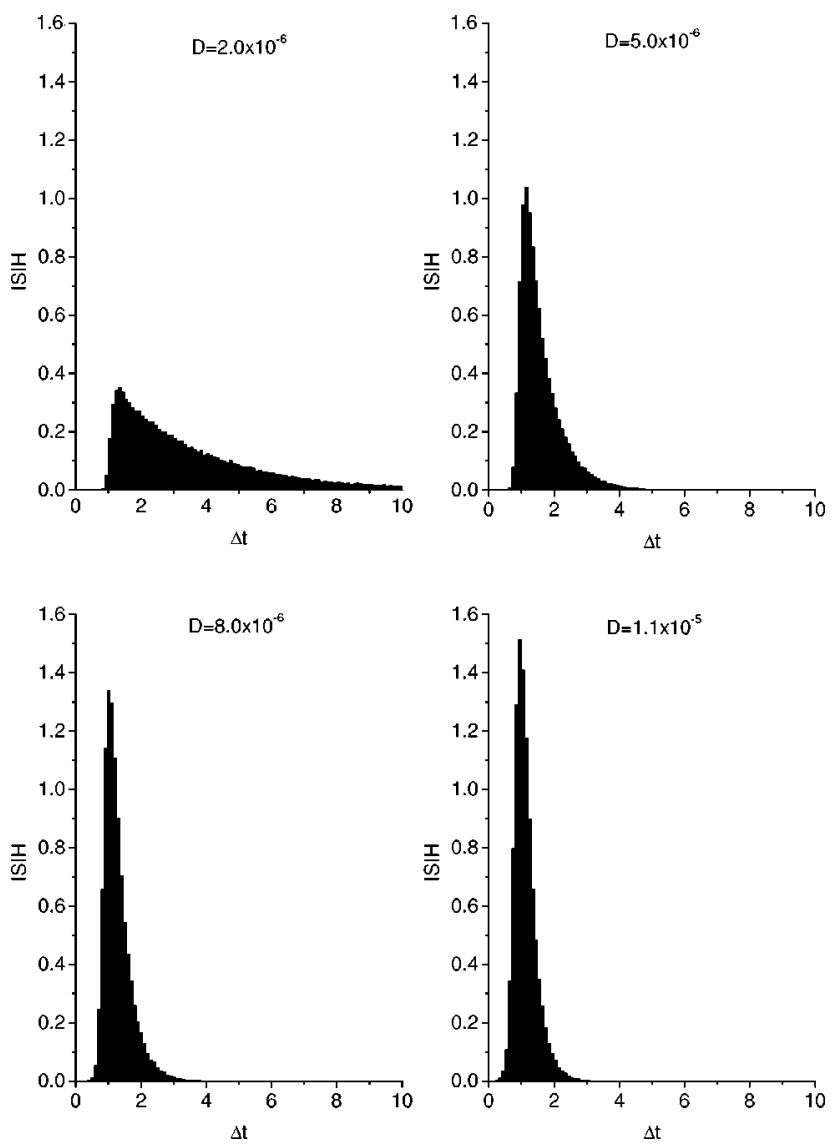

FIG. 2. ISI histograms for several noise intensities $D$ for the pure stochastic FN neuron without external stimulus.

input current that hereafter, and in order to compare with previous results, has been fixed to $I=0.04$ so that noise is the only agent that causes the FN neuron to fire. There are several ways to model the features of the noise being, in terms of Poissonian shot noise [17] or uncorrelated Gaussian white noise, the most frequent. We have considered the last option by assuming a Gaussian random process of intensity $\sqrt{2 D}$. As usual, the response of the system is measured in terms of a series of spikes, $O(t)$, instead of considering the whole evolution of the fast variable $v$. In this way, we get rid of subthreshold oscillations and other time structures that do not convey information. Our analysis is performed by using three different standard measures: the power spectrum of the spike train, the normalized interspike histogram $\operatorname{ISIH}, \varphi$, and the autocorrelation time of the output.

The response strongly depends on the intensity of noise $D$ as we can see from the group of pictures shown below. Figure 1 displays the power spectrum for several noise intensities. For small $D$ the background is almost uniform with no apparent structure. However, as the noise intensity increases, the contribution of intermediate frequencies becomes more important in contrast to the low $\omega$ range, which is much weaker because it is easier to induce firings. The position, height, and width of the broad peak in the power spectrum depends on the noise intensity. Measures of coherence defined in [7] as the product of the height of the peak $H$ to its quality factor $Q$ as

$$
\beta=H Q, \quad Q=\frac{\nu_{p}}{\Delta \nu},
$$




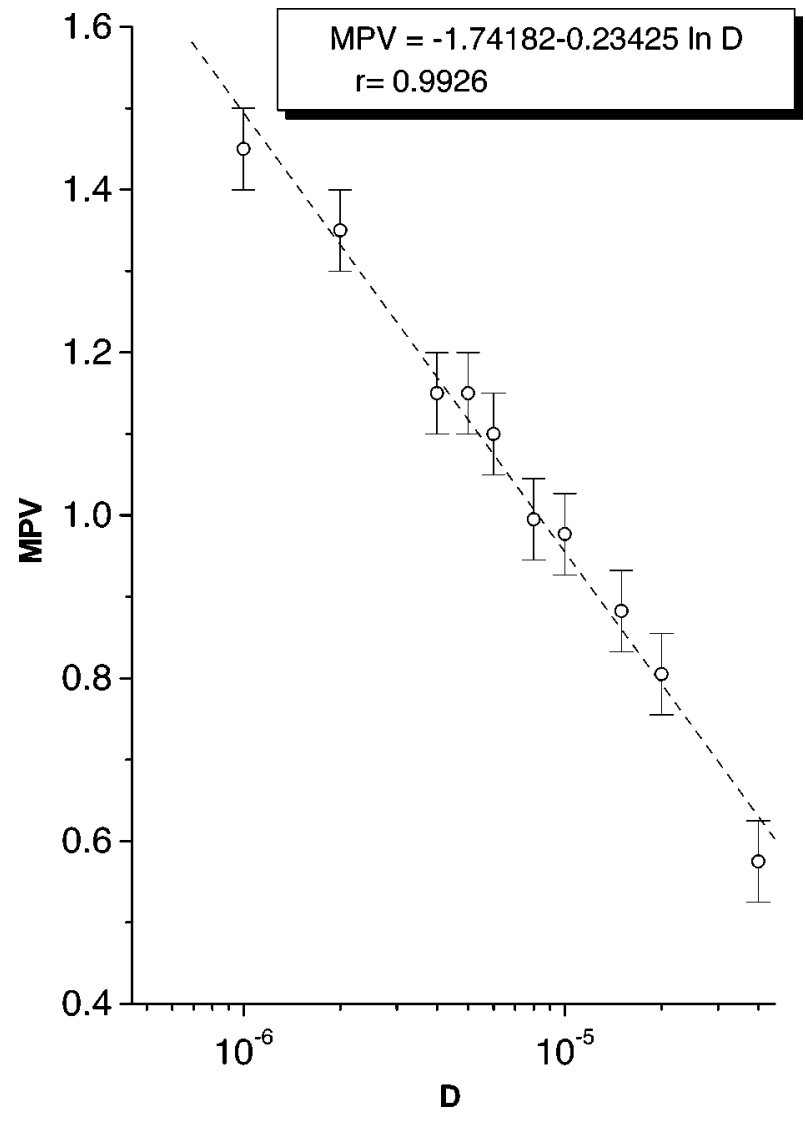

FIG. 3. Most probable value of the ISI histogram for the pure stochastic FN neuron vs the noise intensity.

where $\nu_{p}$ is the frequency of the peak and $\Delta \nu$ is the width at the half-maximum height, are maximized for a noise intensity near $D=8.0 \times 10^{-6}$ (data not shown). For this noise intensity the maximal contribution to the power spectra comes from a region centered around $\nu_{R} \approx 0.85$. Therefore, this first analysis shows the existence of a noise-induced characteristic time scale which causes the system to behave as a stochastic oscillator. This is the fingerprint of the so-called coherent resonance phenomenon (CR) [6]. It is straightforward to think of $\nu_{R}$ as the natural candidate for which a classical resonant process takes place.

Figure 2 shows the ISI distribution for different values of $D$. Now, the different regimes are more evident. For small noise, the distribution is very broad and asymmetric, which confirms the continuous flat power spectrum found in Fig. 1 while for large $D$ the ISIH peak becomes more pronounced. Notice that while the mean value of the distribution is quite variable and changes more than one order of magnitude in the selected range of $D$, the most probable value (MPV) changes very little. A close analysis of the noise dependence of the MPV (see Fig. 3) shows a logarithmic relation with $D$. This fact is important because the MPV turns out to be a very robust quantity that gives information about the internal clock of the system and whose effects remain even in the presence of a signal, as we will see later. It is also responsible for the main peak observed in the power spectrum shown above becoming the representative parameter of the distribution. In the limit of large $D$ the ISI distribution tends to be symmetric and the mean and the MPV tend to the same value.

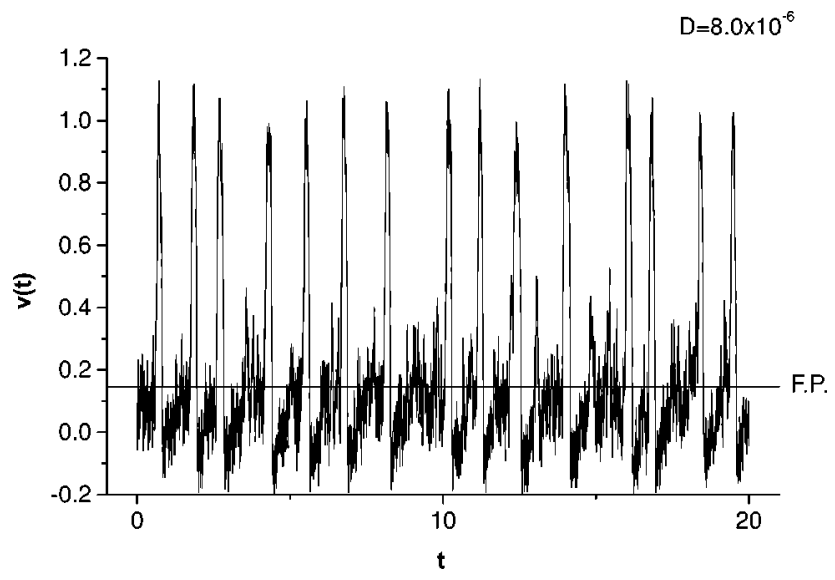

FIG. 4. Excursion time versus activation time for the pure stochastic FN neuron without external stimulus for a noise intensity $D=8.0 \times 10^{-6}$.

Finally, as a complement of the previous discussion let us mention that we have also analyzed the autocorrelation function of the series of spikes following the scheme proposed in [6]. Again a peak for a noise intensity near $D=8.0 \times 10^{-6}$ confirms the existence of the characteristic time scale typical of CR as we could expect from the Wiener-Khinchin theorem. However, we want to point out several comments about this phenomenon. The total period $t_{p}$ between consecutive firings is split in two times: the activation time $t_{a}$ and the
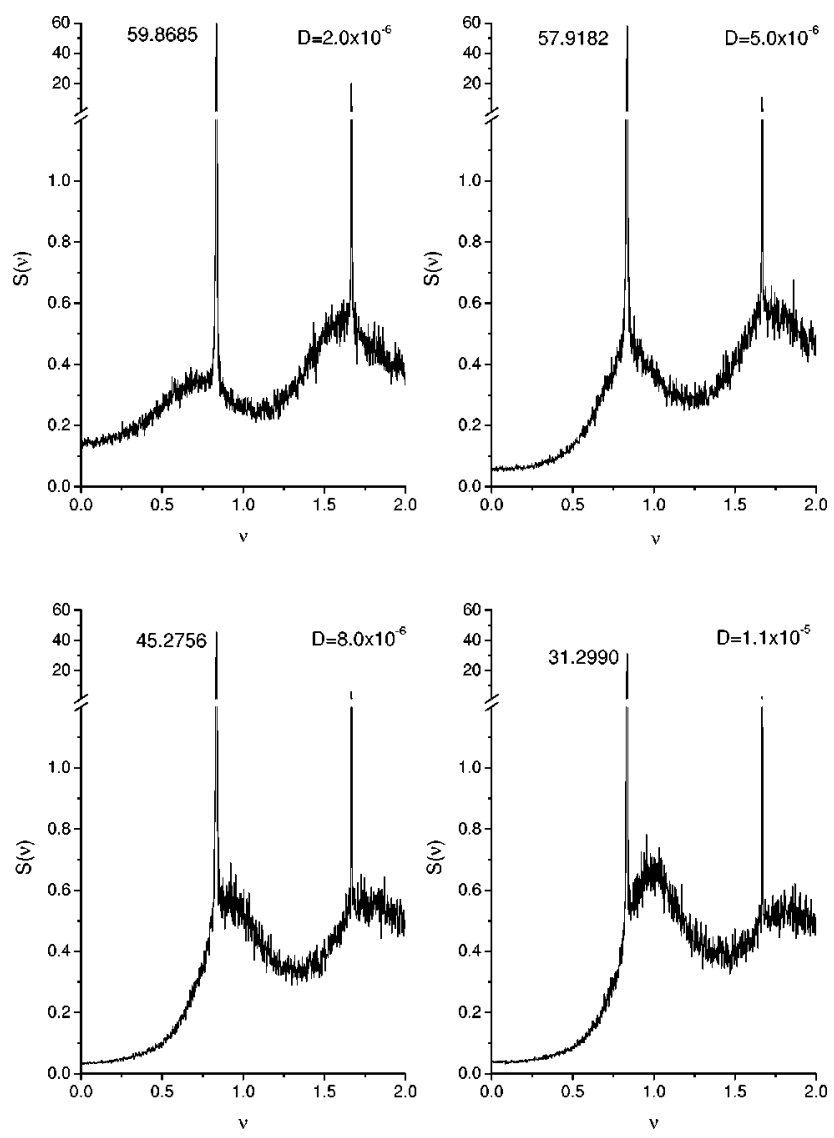

FIG. 5. Power spectrum for several values of $D$ for the stochastic FN neuron when an external sine wave stimulus with period $T$ $=T_{R}$ is applied. 

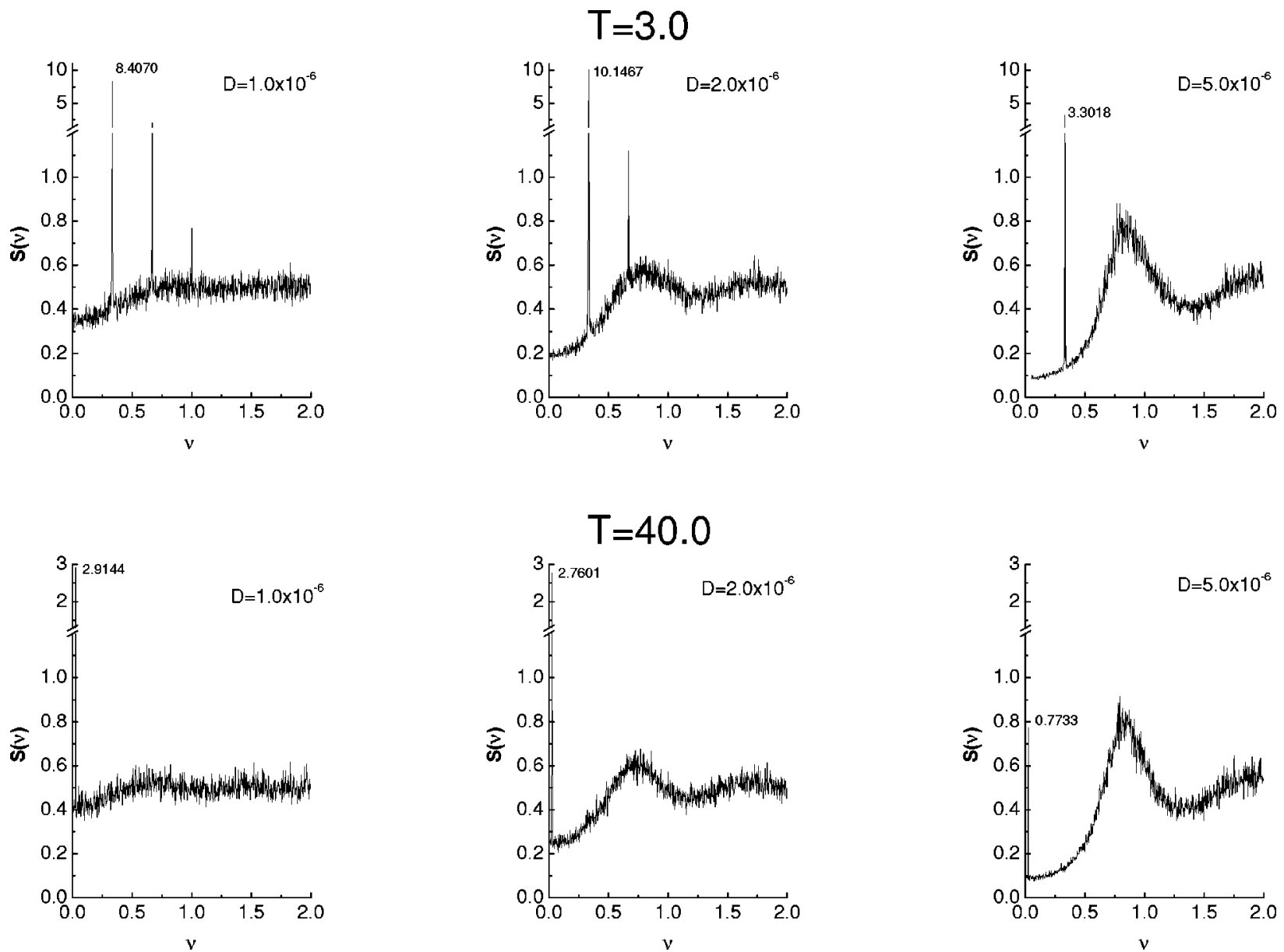

FIG. 6. Power spectrum for several values of $D$ for the stochastic FN neuron when an external sine wave stimulus with period $T=3.0$ (above) and $T=40.0$ (below) is applied.

excursion time $t_{e}$. In [6] a theory is presented, based on several important assumptions. First, that the excursion time is much larger than the activation time, so that a theory for $t_{p}$ can be accounted for by analyzing just the motion of the slow variable, as in [18]. Figure 4 shows that for the range of parameters used in this paper this is a crude approximation since both are of the same order of magnitude $\left(t_{a}\right.$ is even slightly larger than $t_{e}$ ). A possible theoretical alternative is to realize that the ISIH follows an inverse Gaussian distribution [18]

$$
\varphi(t)=\left(\frac{\lambda}{2 \pi}\right)^{1 / 2} t^{-3 / 2} e^{-\lambda(t-\mu)^{2} / 2 \mu^{2} t},
$$

where $\langle\varphi\rangle=\mu, \sigma_{D}^{2}=\mu^{3} / \lambda$, and the MPV, $t_{\mathrm{MP}}=$ $-3 \sigma_{D}^{2} / 2 \mu\left[1-\sqrt{1+\frac{4}{9}\left(\mu / \sigma_{D}\right)^{4}}\right]$. Then, assuming the output of the model as a Markovian shot-noise process and the normalized ISIH as its renewal density, the autocorrelation function of the output $C(\tau)=\langle O(t) O(t+\tau)\rangle$ can be written in the Laplace space as

$$
\bar{C}(s)=\frac{1}{2 \mu}\left[\frac{1+\bar{\varphi}(s)}{1-\bar{\varphi}(s)}\right]-\frac{1}{\mu s}
$$

and by using simple transformations it can be shown that

$$
S(\omega)=2 \operatorname{Re}[\bar{C}(i \omega)]
$$

In this way, it is possible to find all the relevant quantities by using usual tools of stochastic processes. A good review can be found in [19]. On the other hand, it is not clear at all whether CR defines a useful and realistic scenario for signal transmission. In fact, when optimal CR takes place, the ISI distribution is far from being Poissonian and the high regularity displayed in Fig. 2 poses serious doubts about the possible modulation of the firing rate elicited by an external stimulus. This is one of the main issues of the next section.

\section{PERIODIC SIGNAL MODULATION}

Let us discuss some of the open questions raised in the preceding section. Our first goal is to verify the existence of a classical resonant effect. Keeping this goal in mind, we have included a forcing periodic external signal of small subthreshold (in the absence of noise) amplitude and period $T_{R}=1 / \nu_{R} \approx 1.2$. Figure 5 shows again the power spectrum for several values of the noise intensity. Let us remark several points. First, as we could expect, there is a clear nonlinear effect since there is a strong magnification of the contribution of $\nu_{R}$ to $S(\nu)$, in fact, more than two orders of magnitude with respect to the free signal case and one order 

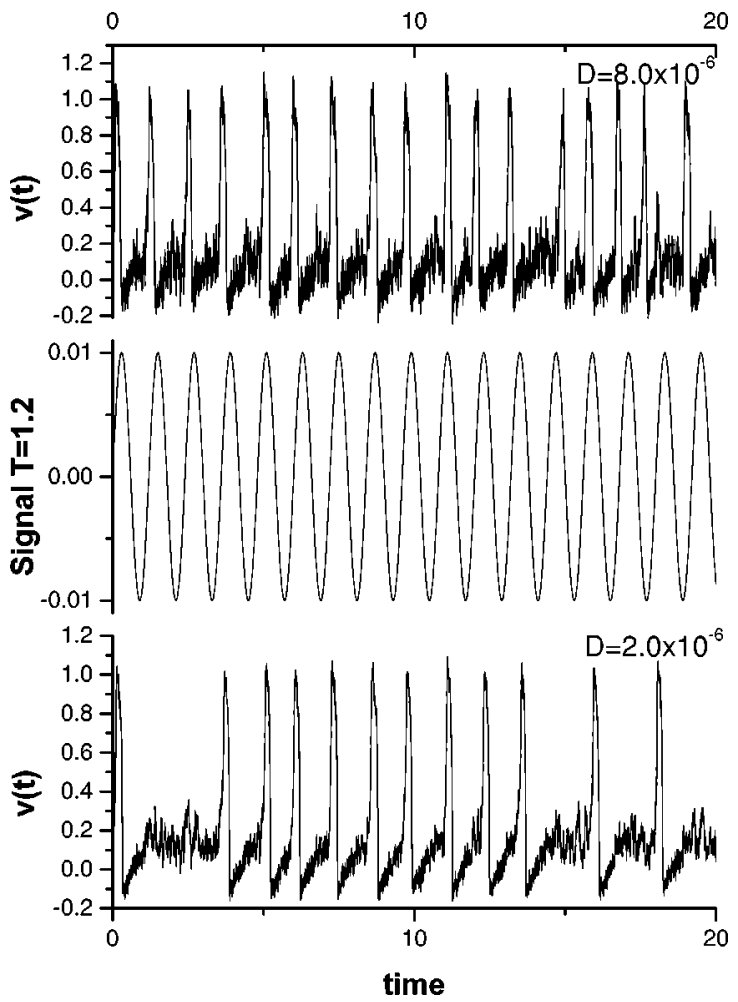

FIG. 7. Temporal evolution of the fast variable $v$ for $D=2.0$ $\times 10^{-6}$ and $D=8.0 \times 10^{-6}$ when the period of the external signal is $T_{R}=1.2$.

of magnitude with respect to any other periodic signal, as we can see in Fig. 6. The resonant effect is observed in a large range of values of $D$, which means that the system is very robust when $T_{R}$ induces its rhythmic response. Moreover, in contrast to typical SR, the magnitude of the resonance is not monotonic with the frequency but there is an optimal value for the frequency of the external input for which the effect is magnified. This fact resembles the typical classical resonance phenomenon observed in deterministic oscillators. The optimal value for the frequency is that of the internal clock when the pure stochastic FN shows the CR effect.

It is interesting to notice that the highest peak in the power spectrum is found for a noise intensity different from that observed for CR in the signal-free case. To understand this fact, which at first glance might seem very surprising, let us look at Figs. 7 and 8, where we have paid special attention to the correlation time between signal and output. In Fig. 7 we can see that while for $D=8.0 \times 10^{-6}$ there is a clear $1: 1$ synchronization, for $D=2.0 \times 10^{-6}$ the system fails to follow the signal quite often. However, according to the power spectrum, the model responds more coherently in the second case. The firing phase histogram shown in Fig. 8 explains this apparent paradox and also sheds some light on the particular structure of the firing pattern. When the optimal CR noise $\left(D=8.0 \times 10^{-6}\right)$ induces the rhythmic activity of the neuron, there is a complete modulation of the signal keeping accurate information about its particular shape, not only in the half positive part of the cycle but also in the negative region. These results are also reflected in the normalized correlation input/output firing rate evaluated as in [9], which has its maximum at the aforementioned value of the noise intensity, see Fig. 8. (Note that in this context, the
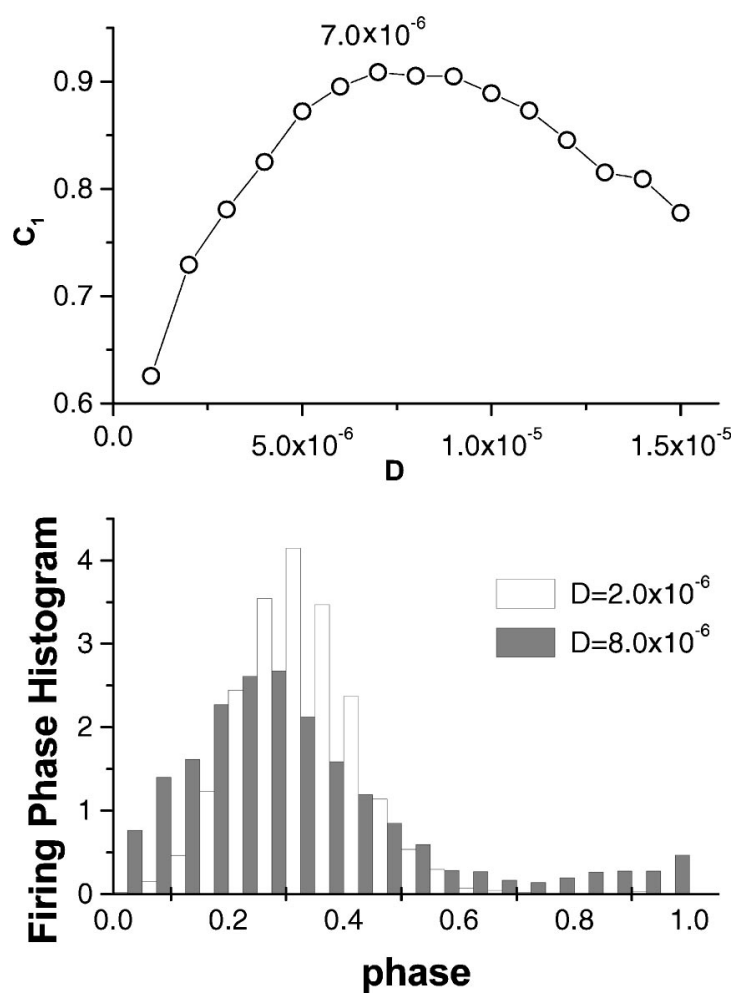

FIG. 8. (Top) Normalized correlation input/output firing rate $C_{1}$ vs the noise intensity $D$. (Bottom) Phase histograms of the series of spikes for $D=2.0 \times 10^{-6}$ and $D=8.0 \times 10^{-6}$. The period of the external signal is $T=1.2$ in both cases.

temporal window used to evaluate the firing rate has to be narrower as the firing rate grows in order to keep highfrequency changes. Therefore, this measure is highly dependent on the width of this window, which should be adjusted to a value of the order of the typical ISI or lower.) It is clear that in the lower noise case we cannot say the same as the output does not carry information about the details of the signal in the half negative part of the periodic forcing. Now, let us consider only the positive half part of the cycle. While in the first case the FN neuron firing is distributed among all the phases following the original shape of the external signal in the second case, this shape is deformed in such a way that a narrow interval which coincides with the maximum value of the periodic stimulus is overvalued. The net effect is that for small $D$ there is a large contribution to the spectrum coming from a reduced set of frequencies while for large $D$ the effect is broadened. From our point of view, this is clear proof that measures of the signal-to-noise ratio (SNR) based only on the height of the peak are misleading when referred to signal information transmission.

Let us make a prediction about the minimal $D$ required to observe firings in the negative part of the cycle, at least in the 1:1 synchronization regime. The condition is fulfilled when the half-period of the signal is of the same order of magnitude as the fluctuations $\sigma_{D}$ of the firing events (width of the ISIH) induced by $D$. If $\sigma_{D}$ is too large, noise dictates the firing times almost independently of the input, and consequently a spike can occur anywhere without signal control. On the other hand, if $\sigma_{D}$ is too small the system becomes very robust and a weak external signal is not able to modulate the firing train enough. It is when $\sigma_{D}$ approximately 

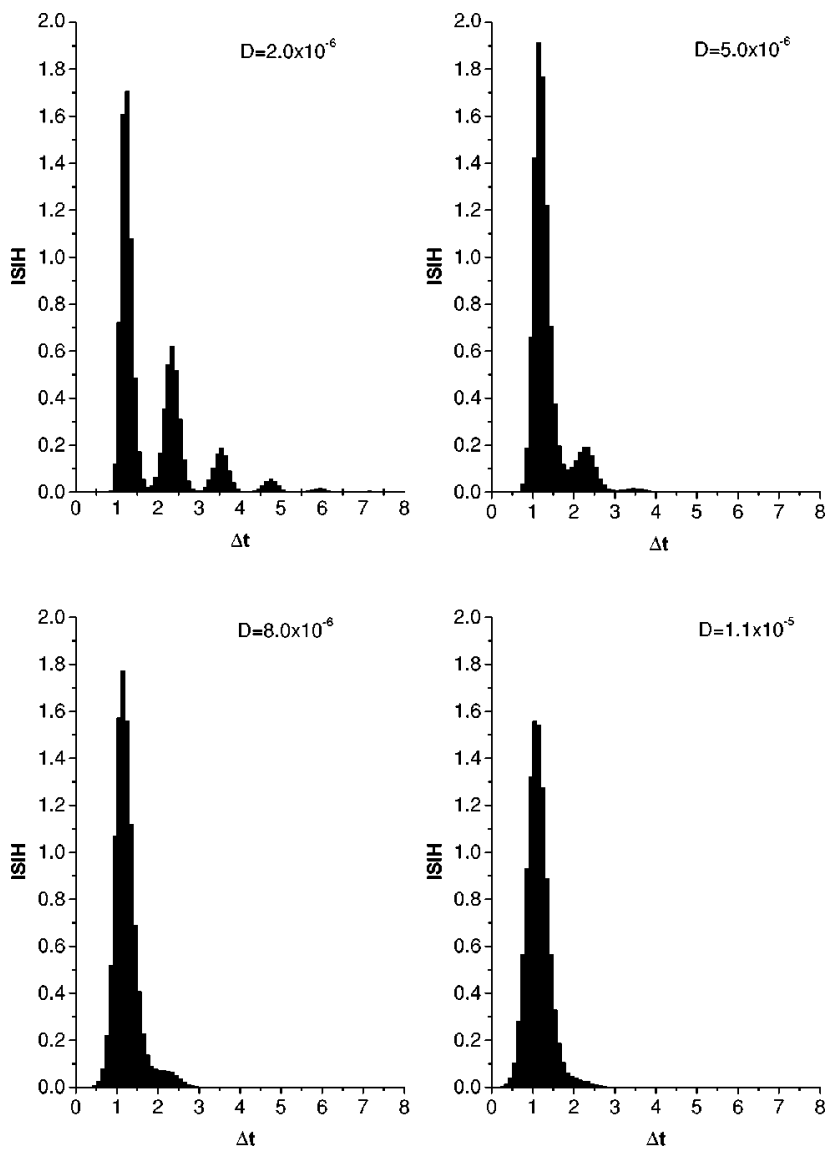

FIG. 9. ISI histograms for several noise intensities $D$ for the stochastic FN neuron when an external stimulus of period $T=T_{R}$ is applied.

matches the half-period of the forcing that the external signal can modulate the firing probability in one period leading to the best possible information transmission. Notice that according to these arguments the optimal input/output correlation depends strongly on the period of the signal and as a consequence there is not just one single optimal value of $D_{T}$; in fact, it is a function of $T$. When analyzing signal-free simulations, the prediction indicates that the noise intensities required to better transmit external signals of different periods are $\left(T=40, D \approx 1.0 \times 10^{-6}\right),\left(T=3, D=2.0 \times 10^{-6}-4.0\right.$ $\left.\times 10^{-6}\right)$, and $\left(T=1.2, D \approx 8.0 \times 10^{-6}\right)$, which are in good agreement with the values observed in Fig. 6 and from firing phase histograms.

In addition, let us remark on another aspect that makes the behavior of the system different from other situations reported in the literature. One of the relevant features of SR is that the best response of the system is reached when the mean output of the unit matches the period of the external signal [8]. In contrast, here, as the MPV of the ISI histogram becomes almost fixed, we can only modulate the magnitude of the fluctuations of the output firing train becoming the relevant parameter of the firing distribution. These results are not in contradiction with those reported in previous papers $[8,9,14,15]$ because one of the most extended assumptions included in any theory about SR is to consider slow signals, which allows us to introduce adiabatic approximations. The ISI distribution is very broad and nearly symmetric in this limit. Then the MPV becomes irrelevant, but these approxi-

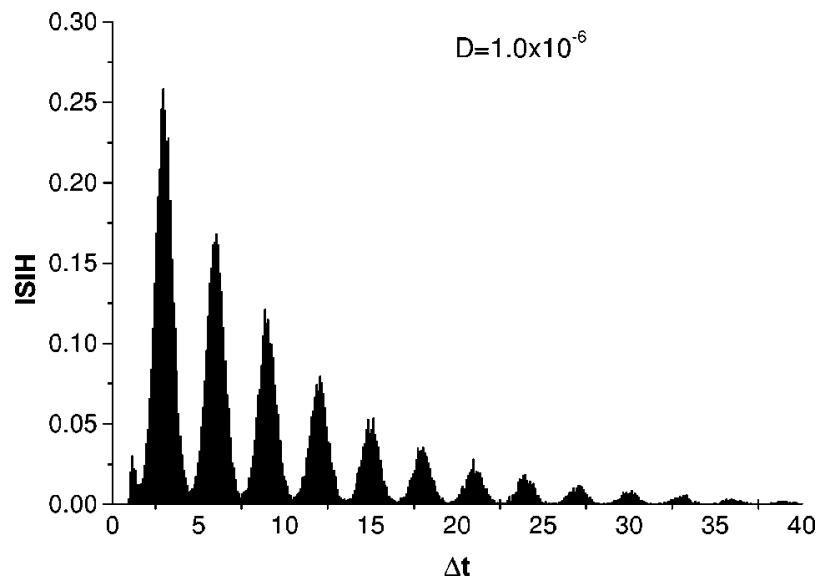

FIG. 10. ISI histogram for a noise intensity $D=1.0 \times 10^{-6}$ for the stochastic FN neuron when an external stimulus of period $T$ $=3$ is applied.

mations are not valid in the high-frequency regime. A different situation may arise for a population of neurons working in parallel, as has been reported in [13]. This study is currently in progress.

We have also analyzed the modulation induced by the presence of the signal from the set of ISI's, see Fig. 9. For small values of $D$ we observe the typical multimodal structure with peaks located at multiples of $T_{R}$. As $D$ increases, such structure fades out leading to a single peak, much narrower than in the free signal case, which displays the onset where 1:1 synchronization takes place. As a consequence, the mean value of the distribution is very sensitive to the action of the external stimulus while the MPV hardly changes, remarking again that the natural period of the system is very robust. Furthermore, there is another difference between this phenomenon and the typical SR. The resonant regime in this case is not reached when the ISI distribution is multimodal but when all the modes collapse in the first one. Measures of the height of the first peak of this distribution also show a maximum for a noise intensity near $D=8.0$ $\times 10^{-6}$.

It is also interesting to analyze another behavior when the system is forced with smaller frequency signals. First of all, we have considered an intermediate value $\nu=\frac{1}{3}$, far enough from $\nu_{R}$ to neglect the nonlinearities induced by the resonant effect but large enough to induce strong modulation in the small $D$ regime. Figure 10 shows the ISIH for $D=10^{-6}$ showing an interesting structure not commented upon previously in the literature and which is almost identical to that observed from a single auditory nerve fiber of a squirrel monkey [10]. In addition to the standard peaks at approximately period multiples of the forcing signal, a new peak of higher frequency comes out which precisely matches the natural period of the system $T_{R}$. Notice that this peak was absent in [10] but not in the original figure coming from experimental data, which suggests that realistic models should give evidence of the existence of its characteristic time even in the subthreshold regime. Such a fingerprint remains except for extremely small noise intensities. The noise intensity required to match the experimental data is lower than that required to better transmit the forcing signal ( $T$ $=3.0$ ). This fact reinforces again the idea that when the 

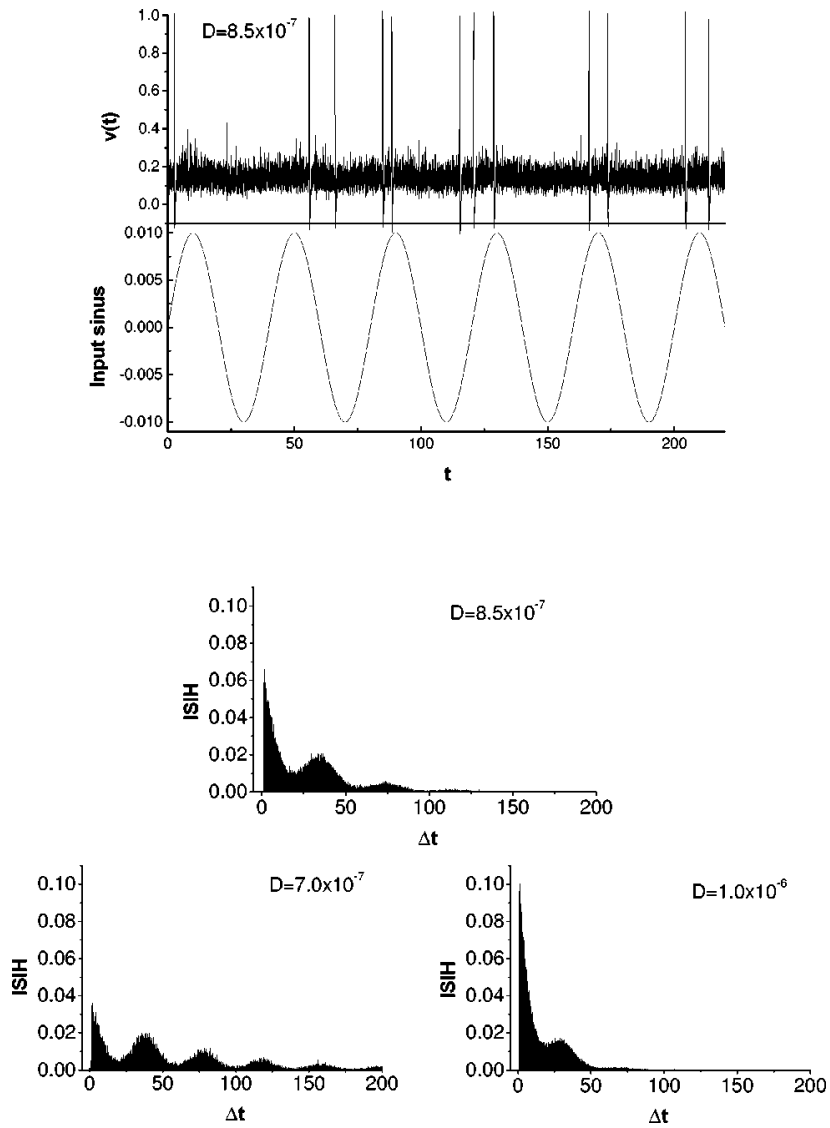

FIG. 11. ISI histogram and temporal evolution of the fast variable $v$ for several values of $D$ around $8.5 \times 10^{-7}$ for the stochastic FN neuron when an external signal of period $T=40$ is applied.

system is driven by the optimal resonant noise, its response is very robust displaying a low degree of variability in the spike train. Therefore, for a real neuron it is convenient to be operated by a lower level of noise in order to offer a variable response when the external signal is changed.

Finally, let us analyze the effect of a low-frequency forcing term, $T=40$, which is much bigger than the natural period of the model but small enough to neglect the adiabatic approximation. In this case the intensity of noise required to get an optimal information transmission is $D \approx 1.0 \times 10^{-6}$. A direct comparison between the ISI mean value and $T$ shows that both are so different, $T /\langle\varphi\rangle>10$, that we are far from the standard SR regime. Indeed, we have observed SR only for much slower signals.
A deeper analysis of the response of the system shows that even in this regime the firing pattern carries information about the intrinsic period $T_{R}$. Figure 11 displays the time evolution of the fast variable $v$ and the ISIH for the previous signal but for a lower noise strength $D=8.5 \times 10^{-7}$ so that we can observe 1:2 synchronization. Close to the maximum of the periodic signal the response of the neuron has a typical structure where two consecutive firings are separated by a short interval which is closely related to $T_{R}$. This time is the most natural choice for the system as we have seen before. The ISIH reflects clearly such structure with two peaks, one centered in $T_{R}$ and the other in $T-T_{R}$. This a very efficient way to get information not only about the features of the external stimulus, but also about the intrinsic response of the system indicating the ISIH is a very good tool. Let us remark that such precise information cannot be obtained either from the firing rate or other measures which use convolutions between the output and long temporal windows where tiny time details are hidden.

\section{CONCLUSIONS}

In this paper we have analyzed the response of a FN neuronlike model to high-frequency external stimuli in the presence of noise. We have seen that the noisy system has a characteristic internal time typical of the coherence resonance phenomenon. It is a fingerprint that remains present in all the time-dependent properties of the model. We have shown that the system displays a classical resonant effect for such a frequency and that depending on the features of the signal there is always an optimal noise strength which maximizes the correlation between input and output, but it has nothing to do with the typical stochastic resonance so extensively studied for the FN model in recent years. Furthermore, we have shown that even for very slow signals, by analyzing the ISI distribution, it is possible to extract information not only about the stimulus but also about $T_{R}$, which can be very helpful when analyzing real time data. Finally, we can reproduce experimental data observed in a single auditory nerve fiber of a squirrel monkey with this kind of analysis.

\section{ACKNOWLEDGMENTS}

We acknowledge financial support from the Spanish DGES through Grant No. PB96-0168. S.R.M acknowledges financial support from Ministerio de Educación y Cultura through a Ph.D. grant No. AP96. Finally, S.R.M. wants to thank Francisco Masanés Landa (1920-1996) for his constant support.
[1] W.R. Softky and C. Koch, J. Neurosci. 81, 334 (1993).

[2] G. Gerstein and B. Mandelbrot, Biophys. J. 4, 41 (1964).

[3] M. Shadlen and W. Newsome, Curr. Biol. 4, 1569 (1994).

[4] D. Amit, Cerebral Cortex, 7, 237 (1997).

[5] M. Stemmler, Network 7, 687 (1996).

[6] A.S. Pikovsky and J. Kurths, Phys. Rev. Lett. 78, 775 (1997).
[7] G. Hu, T. Ditzinger, C.Z. Ning, and H. Haken, Phys. Rev. Lett. 71, 807 (1993).

[8] L. Gammaitoni, P. Hänggi, P. Jung, and F. Marchesoni, Rev. Mod. Phys. 70, 223 (1998).

[9] J.J. Collins, C.C. Chow, and T.T. Imhoff, Phys. Rev. E 52, R3321 (1995). 
[10] A. Longtin, Phys. Rev. Lett. 67, 656 (1991) and Ref. [4] therein.

[11] R. Fitzhugh, Biophys. J. 1, 445 (1961).

[12] J.S. Nagumo, S. Arimoto, and S. Yoshizawa, Proc. IRE 50, 2061 (1962).

[13] J.J. Collins, C.C. Chow, and T.T. Imhoff, Nature (London) 376, 236 (1995).

[14] D.R. Chialvo, A. Longtin, and J. Muller-Gerking, Phys. Rev. E 55, 1798 (1997).
[15] A. Longtin and D.R. Chialvo (unpublished).

[16] M.V. Tsodyks and T. Sejnowsky, Network 6, 111 (1995).

[17] F. Chapeau-Blondeau, X. Godivier, and N. Chambet, Phys. Rev. E 53, 1273 (1996); X. Godivier and F. ChapeauBlondeau, Europhys. Lett. 35, 473 (1996).

[18] J. Grasman, Asymptotic Methods for Relaxation Oscillations and Applications (Springer-Verlag, New York, 1987).

[19] Josep M. Porrà, Ph.D. thesis, Universitat de Barcelona, Spain (1993). 